\title{
FATORES ASSOCIADOS A PRÁTICA DE ATIVIDADE FÍSICA EM IDOSOS USUÁRIOS DE ACADEMIA DA TERCEIRA IDADE DE MARINGÁ-PR
}

Article · December 2017

DOI: $10.5902 / 2236583424800$

CITATIONS

0

5 authors, including:

Daniel Vicentini de Oliveira

Faculdade Metropolitana de Maringá

54 PUBLICATIONS 11 CITATIONS

SEE PROFILE

Jose Roberto Andrade do Nascimento Junior

Universidade Federal do Vale do São Francisco (UNIVASF)

104 PUBLICATIONS 86 CITATIONS

SEE PROFILE
READS

82

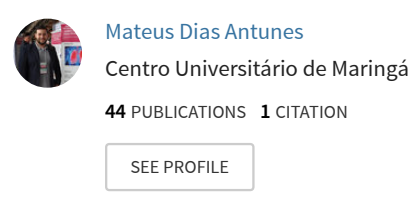

Some of the authors of this publication are also working on these related projects:

Estudo do desenvolvimento das variáveis psicológicas e comportamentais de atletas jovens e adultos do estado de Pernambuco, Brasil. View project

Análise da Importância do Envolvimento na Atividade Física, nos Hábitos Alimentares e nos Comportamentos Sedentários de Adolescentes e Jovens Escolares com Estatutos Socioeconômicos Diferenciados View project 


\section{FATORES ASSOCIADOS A PRÁTICA DE ATIVIDADE FÍSICA EM IDOSOS USUÁRIOS DE ACADEMIA DA TERCEIRA IDADE DE MARINGÁ-PR}

\section{FACTORS ASSOCIATED WITH THE PRACTICE OF PHYSICAL ACTIVITY IN ELDERLY USERS OF THE MARINGÁ-PR'S ELDERLY ACADEMY}

Daniel Vicentini de Oliveira, Priscila Martins Peres, Mateus Dias Antunes, Maura Fernandes Franco, José Roberto Andrade do Nascimento Júnior

\section{RESUMO}

Este estudo teve o objetivo de investigar a associação do nível de atividade física com as variáveis sociodemográficas e de saúde, a capacidade funcional e a força muscular de idosos. Participaram deste estudo transversal 73 idosos fisicamente ativos, usuários das Academias de Terceira Idade do município de Maringá/PR. Os instrumentos utilizados foram o International Physical Activity Questionnaire, a Escala de Latws, Escala de Lawton e os testes Flexão de cotovelo e Levantar e sentar. Houve correlação das atividades básicas com as instrumentais $(r=0,26)$ e com a força muscular de membro inferior $(r=0,25)$, além de relação entre a força muscular de membro superior com a de membro inferior $(r=0,44)$. Conclui-se que idosos praticantes de atividade física (independente do nível) apresentam boa capacidade funcional. Entretanto, aqueles com maior força muscular apresentam maior independência funcional.

Descritores: força muscular, atividade física, idoso, promoção da saúde

\section{ABSTRACT}

This study aimed to investigate the association of physical activity level with sociodemographic and health variables, functional capacity and muscle strength among elderly. This cross-sectional study was carried out with 73 elderly, users of the Third Age Academies in the city of Maringá, Paraná State. The instruments were the International Physical Activity Questionnaire, the Latws Scale, the Lawton Scale, and the Elbow Flexion and Lift and Sit tests were used. There was a correlation between basic and instrumental activities $(r=0.26)$ and lower limb muscle strength $(r=0,25)$, as well as the relationship between upper and lower limb muscle strength $(r=0,44)$. It is concluded that elderly people practicing physical activity (regardless of level) have good functional capacity. However, those with greater muscle strength have greater functional independence.

Descriptors: muscle strength, motor activity, aged, health promotion

Daniel Vicentini de Oliveira, Doutorando em Gerontologia pela Universidade Estadual de Campinas (UNICAMP), Campinas, SP, Brasil.

Priscila Martins Peres, Graduada em Educação fisica pela Faculdade Metropolitana de Maringá (FAMMA), Maringá, Paraná, Brasil.

Mateus Dias Antunes, Mestrando em Promoção da Saúde pelo Centro Universitário Cesumar (UNICESUMAR), Maringá, Paraná, Brasil.

Maura Fernandes Franco, Mestranda em Gerontologia pela Universidade Estadual de Campinas (UNICAMP), Campinas, SP, Brasil..

José Roberto Andrade do Nascimento Júnior, Doutor em Educação fisica pela Universidade Estadual de Maringá (UEM), Maringá, PR, Brasil. 
O envelhecimento humano é um processo marcado por alterações fisiológicas ${ }^{1}$, que ocorrem de maneira diferenciada, em maior ou menor intensidade, em todos os sistemas ${ }^{2}$. No sistema musculoesquelético, algumas alterações são evidentes como a perda de massa muscular e redução da força muscular fatores que, por decorrência3, determinam são evidentes como a perda de massa muscular e redução da força muscular
limitação da capacidade de coordenação e de controle do equilibrio corporal ${ }^{4}$.

Além da redução da força muscular, ocorrem também alterações na capacidade de coordenação, deterioração da elasticidade e estabilidade dos músculos, tendões e ligamentos, além da redução da flexibilidade, promovendo danos nas articulações 5,6 . Souto et al. ${ }^{7}$ afirmam que a redução da força está relativamente associada à sarcopenia, seja por atrofia, ou diminuição do número de fibras musculares. Os mesmos ainda apontam que a diminuição da força em membros inferiores predomina-se com $o$ avanço da idade em relação aos membros superiores.

A força muscular está intimamente relacionada com a capacidade funcional do idoso $0^{5}$, que é compreendida como a capacidade de realização das atividades básicas de vida diária (ABVDs) e as atividades instrumentais de vida diária (AIVDs) de maneira eficaz, com segurança e sem sentir cansaço excessivo8. Entretanto, como já salientando anteriormente, o processo do envelhecimento pode diminuir a capacidade de gerar essa força, comprometendo de forma parcial ou total a realização dessas atividades, aumentando o risco de quedas, fraturas e diminuição da sobrevida ${ }^{5}$.

Em suma, a diminuição de força muscular pode desenvolver restrições como a redução da velocidade da marcha, dificuldade de subir escadas, conduzir objetos, entre outras. Por outro lado, este comprometimento muscular prejudica, sobretudo, os idosos fisicamente inativos ${ }^{9}$. Com base neste cenário, considera-se fundamental a prática de atividades físicas ao longo da vida 4 .

As práticas de atividades físicas são benéficas à população idosa, pois melhora a qualidade de vida e a capacidade funcional e, com isso, reduz os riscos de doenças e incapacidades ${ }^{4,10}$. Além disso, manter um comportamento ativo pode beneficiar a população idosa nos aspectos biológicos, psicológicos e sociais melhorando a qualidade de vida ${ }^{11}$.

Diante do exposto, o presente estudo teve como objetivo investigar a associação do nível de atividade física com as variáveis sociodemográficas e de saúde, a capacidade funcional e a força muscular de idosos usuários das Academias de Terceira Idade do município de Maringá/PR.

Metodologia

Trata-se de um estudo quantitativo, observacional e transversal, aprovado pelo Comitê de ética em Pesquisa com seres humanos do Centro Universitário Cesumar por meio do parecer número 1694862/2016.

Foram incluídos idosos de ambos os sexos, usuários das Academias de terceira idade (ATIs) há no mínimo três meses e com frequência de duas vezes semanais. Os idosos foram recrutados por conveniência em seis ATIs localizadas nas regiões centrais do município de Maringá, pois estas ATIs são as mais frequentadas por idosos, porque o município consta com 54 ATIs espalhadas em diversos bairros. Maringá é $07^{\circ}$ maior município de Sul do País, localizada no noroeste do Paraná. 0 município de Maringá consolidou-se como um importante polo regional. Atualmente é a terceira maior cidade do Paraná e $066^{\circ}$ município mais populoso do país.No último censo disponível, em 2010, a população total em Maringá era de 357.077 habitantes e destes, de idosos era de $43.353(12,1 \%)$.

Foram excluídos idosos usuários de acessórios para marcha (bengala, andador, dentre outros) e cadeirantes; idosos praticantes de alguma modalidade de exercício físico; idosos com disfunções visuais, auditivas e cognitivas incapacitantes para a realização dos testes e questionários. A amostra foi escolhida de forma intencional e por conveniência, totalizando 73 idosos, sendo 34 mulheres e 39 homens.

Para caracterização dos idosos foi utilizado um questionário sociodemográfico e de condições de saúde dos idosos, com questões dicotômicas referentes à idade, sexo, raça, escolaridade, tabagismo, aposentadoria, auto percepção de saúde, situação ocupacional, renda em salário mínimo, situação conjugal, quantidade de medicamentos utilizados, presença de doenças, histórico de quedas dos últimos seis meses, tempo de prática nas ATIs e frequência semanal.

Para avaliação do nível de atividade física, foi utilizado o International Physical Activity Questionnaire (IPAQ) versão curta, com questões relacionadas às atividades físicas de intensidade leve, moderada e vigorosa, classificando como sedentário, irregularmente ativo, ativo e muito ativo, de acordo com os dias da semana e o tempo total gasto po dia12. Após a aplicação e classificação do questionário, os idosos foram classificados

A força muscular de membro inferior foi avaliada por meio do Teste Levantar e Sentar da Cadeira em 30 segundos Para tal, foi utilizado um cronômetro, uma cadeira com encosto e sem apoio de braço, com altura de aproximadamente 43 $\mathrm{cm}$. Durante o procedimento o participante mantinha os braços cruzados com o dedo médio em direção ao acrômio. Ao sinal do avaliador o participante ficava na posição totalmente em pé, e em seguida retornava a posição inicial, sendo que repetiu os movimentos quanto foi possível durante 30 segundos. A pontuação foi obtida pelo número total de repetições corretas em 30 segundos 13

O Teste de Flexão de Cotovelo foi utilizado para avaliar a força muscular de membros superiores. Para realização do mesmo foi utilizado um cronômetro, uma cadeira com encosto e sem apoio de braço, halteres de mão (2 kg para mulheres e $4 \mathrm{~kg}$ para homens). 0 participante se posicionou sentado na cadeira com as costas retas, os pés no chão o lado dominante do corpo próximo à borda da cadeira. Segurando o halter com a mão dominante, utilizando uma empunhadura de aperto de mão. 0 teste iniciou-se com o braço estendido próximo a cadeira, perpendicular ao chão. Ao sinal, o participante flexionou o braço em amplitude total de movimento e retornou à posição inicial com o braço totalmente estendido. 0 avaliado executou quantas repetições foram possiveis em 30 segundos 13 .

Acapacidade funcional para as ABVDs foi avaliada por meio da Escala de Katz com questionamentos relacionados ao banho, vestuário, higiene pessoal, transferência, continência e alimentação. Os resultados foram obtidos por meio de pontuação, sendo seis pontos classificados como independência; cinco, quatro ou três pontos, dependência parcial; dois ou menos pontos, dependência total14

Já para as AIVDs foi utilizada a Escala de Lawton, que permite avaliar atividades necessárias para viver de forma independente na comunidade, referentes a telefonemas, viagens, compras, preparo de refeições, trabalho doméstico, medicações e dinheiro. A pontuação final resultou da soma da pontuação das 7 AIVDs e variou entre 0 a 7 pontos correspondendo ao número de AIVD em que o idoso é independente14.

A coleta de dados foi iniciada após a autorização do responsável pelas ATls na Secretaria de Esportes e Lazer do município de Maringá, estado do Paraná. Foram sorteadas cinco ATIs localizadas pelas cinco regiões da cidade (norte, sul, leste, oeste, sudeste) a fim de caracterizar a amostra Maringaense. Os idosos foram abordados nas próprias ATIs em diferentes períodos. Os mesmos foram orientados quanto às justificativas e objetivos do trabalho. Aqueles que aceitaram participar assinaram o Termo de Consentimento Livre e Esclarecido (TCLE).

A análise dos dados foi realizada por meio do Software SPSS 22.0. Foi realizada a análise descritiva e inferencial. Foi utilizado frequência e percentual como medidas descritivas para as variáveis categóricas. Para as variáveis numéricas, inicialmente foi verificada a normalidade dos dados por meio do teste Kolmogorov-Smirnov. Como os dados não apresentaram distribuição normal foram utilizadas Mediana (Md) e Quartis (Q1; Q3) para a caracterização dos resultados. Na comparação entre os grupos (Irregularmente Ativo e Ativo/Muito Ativo), foi utilizado o teste "U" de Mann-Whitney. $O$ teste de Qui-quadrado de Pearson (X2) foi utilizado para se observar as possíveis associações existentes das variáveis sociodemográficas e das condições de saúde com o nível de atividade física. A Regressão Logística binária (análise bruta e ajustada) foi usada para examinar a magnitude das associações das variáveis sociodemográficas e das condições de saúde (variáveis independentes) com o nível de atividade física muito ativo/ativo (variável dependente). Para modelagem da análise de regressão foram consideradas apenas as variáveis que apresentaram nível de significância igual ou inferior a 0,20 no teste do Qui-quadrado, as quais foram inseridas simultaneamente em um único bloco, sem retirada após análise Cada variável exploratória que apresentou valor $p<0,05$ no teste Wald foi considerada como associada ao desfecho do estudo. $\mathrm{O}$ ajuste do modelo foi verificado por meio do teste de Hosmer-Lemeshow. Para verificar a correlação entre a força muscular e a capacidade funcional, efetuou-se o coeficiente de correlação de Spearman. Considerou-se um nível de significância de $p<0,05$.

\section{RESULTADOS:}

Conforme os resultados da Tabela 1, foi encontrada associação significativado nível de atividade física apenas com a situação conjugal $(p=0,008)$, indicando que existe uma maior proporção de idosos irregularmente ativos que são casados $(82,1 \%)$ 
Tabela 1. Associação do nível de atividade física com as variáveis sociodemográficas dos idosos fisicamente ativos de Maringá-PR, 2016.

\begin{tabular}{|c|c|c|c|c|}
\hline \multirow[t]{2}{*}{ VARIÁVEIS } & \multicolumn{2}{|c|}{ Nível de atividade física } & \multirow[t]{2}{*}{$X^{2}$} & \multirow[t]{2}{*}{$\mathrm{P}$} \\
\hline & $\begin{array}{c}\text { Muito ativo/Ativo } \\
f(\%)\end{array}$ & $\begin{array}{c}\text { Irregularmente ativo } \\
f(\%)\end{array}$ & & \\
\hline \multicolumn{5}{|c|}{ 然 } \\
\hline Feminino & $22(64,8)$ & $12(35,2)$ & & \\
\hline Masculino & $17(43,5)$ & $22(56,5)$ & 2,812 & 0,072 \\
\hline \multicolumn{5}{|l|}{ Raça } \\
\hline Caucasiana & $40(88,9)$ & $27(96,4)$ & 2,710 & 0,258 \\
\hline Negra & $4(8,9)$ & $0(0,0)$ & & \\
\hline Asiática & $1(2,2)$ & $1(3,6)$ & & \\
\hline \multicolumn{5}{|l|}{ Faixa etária } \\
\hline 60 a 70 anos & $26(57,8)$ & $15(53,6)$ & 0,124 & 0,725 \\
\hline Mais de 70 anos & $19(42,2)$ & $13(46,4)$ & & \\
\hline \multicolumn{5}{|l|}{ Situação conjugal } \\
\hline Casado & $23(51,1)$ & $23(82,1)$ & 7,132 & 0,008 \\
\hline Não-casado & $22(48,9)$ & $5(17,9)$ & & \\
\hline \multicolumn{5}{|l|}{ Escolaridade } \\
\hline Analfabeto/ Fund. Incompleto & $19(42,2)$ & $12(42,9)$ & 0,131 & 0,717 \\
\hline Fundamental completo & $9(20,0)$ & $3(10,7)$ & & \\
\hline \multirow{2}{*}{\multicolumn{5}{|c|}{ Renda Mensal }} \\
\hline & & & & \\
\hline Menos de 1 salário mínimo & $5(11,1)$ & $2(7,1)$ & & \\
\hline 1 a 2 salários mínimos & $26(57,8)$ & $15(53,6)$ & 0,665 & 0,415 \\
\hline 2 ou mais salários mínimos & $14(31,1)$ & $11(39,3)$ & & \\
\hline \multicolumn{5}{|l|}{ Situacão Ocupacional } \\
\hline Ativo & $14(31,1)$ & $9(32,1)$ & 0,009 & 0,926 \\
\hline Inativo & $31(68,9)$ & $19(67,9)$ & & \\
\hline \multicolumn{5}{|l|}{ Aposentadoria } \\
\hline Não & $12(26,7)$ & $5(17,9)$ & 0,750 & 0,387 \\
\hline Sim & $33(73,3)$ & $23(82,1)$ & & \\
\hline
\end{tabular}

A Tabela 2 apresenta a associação do nível de atividade física com as variáveis relacionadas à saúde dos idosos praticantes de atividade física nas ATIs. Não foi encontrada associação significativa $(p>0,05)$ do nível de atividade física com nenhuma das variáveis relacionadas à saúde dos idosos praticantes de atividade física nas ATIs.

Tabela 2. Associação do nível de atividade física com as variáveis relacionadas à saúde dos idosos fisicamente ativos de Maringá-PR, 2016

$$
\text { VARIÁVEIS }
$$

$$
\text { Nível de atividade física }
$$

$$
\begin{array}{cc}
\text { Muito ativo/Ativo } & \text { Irregularmente ativo } \\
f(\%) & f(\%)
\end{array}
$$

Percepção de saúde

\begin{tabular}{lcccc} 
Boa & $33(73,3)$ & $20(71,4)$ & 0,031 & 0,859 \\
Regular/Ruim & $12(26,7)$ & $8(28,6)$ & & \\
\hline
\end{tabular}

$\begin{array}{lrrrr}\text { Quantidade de Medicamentos } & & & & \\ \text { Não usa medicamento } & 15(33,3) & 4(14,3) & 1,756 & 0,185 \\ 1 \text { tipo } & 19(42,2) & 16(57,1) & & \\ 2 \text { tipos ou mais } & 11(24,5) & 8(28,6) & & \\ \text { Quantidade de doenças } & & & & \\ \text { Não tem doença } & 30(66,7) & 18(64,3) & & \\ 1 \text { doença } & 13(28,9) & 9(32,1) & 0,012 & 0,912 \\ 2 \text { ou mais doenças } & 2(4,4) & 1(3,6) & & \\ \text { Quedas nos últimos 6 meses } & & & & \\ \text { Sim } & 5(11,1) & 2(7,1) & 0,314 & 0,700 \\ \text { Não } & 40(88,9) & 26(92,9) & & \\ \text { Tempo de prática } & & & & \\ 3 \text { meses a 1 ano } & 13(28,9) & 8(28,6) & 2,485 & 0,115 \\ \text { 1,1 a 5 anos } & 8(17,8) & 14(50,0) & & \\ \text { Mais de } 5 \text { anos } & 24(53,3) & 6(21,4) & & \\ \text { *Associação significativa - } p<0,05: \text { Teste de Qui-quadrado para proporções. } & & \end{array}$

A Tabela 3 apresenta a associação do nível de atividade física com a capacidade funcional e força muscular dos idosos praticantes de atividade física nas ATIs. Não foi encontrada associação significativa $(p>0,05)$ do nível de atividade física com nenhuma das variáveis relacionadas à capacidade funcional e à força muscular dos idosos praticantes de atividade física nas ATIs, indicando que o nível de atividade física não é um elemento interveniente no desenvolvimento destas variáveis.

Tabela 3. Associação do nível de atividade física com a capacidade funcional e força muscular dos idosos praticantes de atividade física nas ATIs de Maringá-PR, 2016.

$$
\text { VARIÁVEIS }
$$$$
\text { Nível de atividade física }
$$$$
\text { Muito ativo/Ativo Irregularmente ativo }
$$

$$
f(\%) \quad f(\%)
$$

\section{ABVDs (escore)}

Independência

Dependência Parcial

$42(93,3)$

$3(6,7)$

$26(92,9)$

AlvDs (escore)

Independência
Dependência Parcial/Total

$27(60,0)$

$18(40,0)$

$20(71,4)$

$8(28,6)$

FMMSs (repetições)

Muito Fraco/Fraco

$20(44,4)$

$10(22,3)$

$15(33,3)$

$9(32,1)$

$9(32,1)$

$10(35,8)$

Bom/Muito Bom

FMMls (repetições)

$25(55,6)$

$13(46,4)$

$12(26,7)$
$8(17,7)$

$10(35,7)$

$5(17,9)$

$0,006 \quad 0,997$

Bom/Muito Bom

ABVDs: atividades básicas de vida diária AlVDs: atividades instrumentais de vida diária FMMSs: força muscular de membros superiores; FMMls: força muscular de membros inferiores.

Para modelagem da análise de regressão logística foram consideradas apenas as variáveis que apresentaram nível 
de significância igual ou inferior a 0,20 no teste de Qui-quadrado. Na análise bruta (Tabela 4), verificou-se associação significativa do nível de atividade física muito ativo/ativo apenas com a situação conjugal $(p=0,010)$, evidenciando que os idosos que não são casados possuem 4,4 [IC95\% = 1,421-13,621] vezes mais chances de serem ativos fisicamente quando comparados com os idosos casados.

Tabela 4 - Razão de chances (odds ratio) bruta e ajustada para o nível de atividade física muito ativo/ativo e fatores associados dos idosos praticantes de atividade física nas ATIs de Maringá-PR, 2016.

VARIÁVEIS

Situação conjugal

Casado

Não-casado

Uso de Medicamentos

Não usa medicamento

1 tipo

2 tipos ou mais

Tempo de prática

3 meses a 1 ano

1,1 a 5 anos

Mais de 5 anos

OR bruta(IC 95\%)

p

OR ajustada (IC 95\%)

1,00

$4,400[1,421-13,621]$

$0,010^{*}$

1,00

$0,317[0,087-1,148]$

0,080

0,169

$4,826[1,375-16,937]$

$0,014^{*}$

$0,367[0,088-1,533]$

1,00

$0,285[0,067-1,220] \quad 0,091$

$0,477[0,091-2,492] \quad 0,380$

1,00

$0,346[0,087-1,373] \quad 0,131$

0,160

$2,893[0,681-12,283]$

0,150

$\mathrm{OR}=$ Odds Ratio; $\mathrm{IC}=$ Intervalo de Confiança.

Quando a análise foi ajustada por todas as variáveis do modelo (Tabela 4), somente a situação conjugal se mostrou associada significativamente com o nível de atividade física ativo/muito ativo $(p=0,014)$. Ressalta-se que os idosos que não são casados têm aproximadamente 5 [IC95\% $=1,375-16,937]$ vezes mais chances de serem ativos fisicamente quando comparados aos idosos casados.

A Tabela 5 apresenta a correlação entre a força muscular e a capacidade funcional dos idosos

Tabela 5. Correlação entre a força muscular e a capacidade funcional dos idosos de Maringá-PR, 2016.

VARIÁVEIS ABVDS AIVDS FMMIS FMMSS

$\begin{array}{llll}\text { 1. ABVDs - } \quad 0,26^{*} & 0,25^{*} & 0,21\end{array}$

$\begin{array}{lll}2 . & \text { AlVDs - } \quad-\quad 0,22 & 0,04 \\ 3 . & - & -0,44^{*}\end{array}$

4. FMMS

4. FMMSs - $\quad-\quad-$

${ }^{*}$ Correlação significativa $(p<0,05)$ - Correlação de Spearman. Nota: ABVDs: atividades básicas de vida diária; AIVDs: atividades instrumentais de vida diária; FMMSs: força muscular de membros superiores; FMMls: força muscular de membros inferiores.

Verificou-se uma correlação positiva e significativa $(p<0,05)$ das ABVDs com as atividades instrumentais $(r=$ $0,26)$ e com o teste de sentar e levantar $(r=0,25)$, indicando uma relação fraca entre 0 aumento da capacidade funcional e 0 aumento da força muscular de membros inferiores. Houve correlação significativa também entre o teste de flexão de braços e o teste de sentar e levantar $(r=0,44)$, evidenciando uma relação moderada entre 0 aumento da força muscular de membros inferiores e superiores.
Discussão

Apesar de este estudo não ter separado as análises dos dados por sexo, a literatura atual tem demonstrado um maior predomínio de mulheres praticantes de atividade física. Esse resultado pode indicar três aspectos importantes do envelhecimento ativo: maior longevidade das mulheres 15 , maior adesão delas aos programas de atividade física16 e maior capacidade para mudança de comportamento e de estilo de vida do que os homens17.

Outros estudos 17-18 também evidenciaram em relação à idade dos idosos que praticavam atividades físicas, um predomínio de idosos com menos de 70 anos de idade. As evidências indicam que o NAF tende a reduzir com o passar dos anos, atingindo níveis mais baixos principalmente no grupo dos idosos mais velhos 19 , associando-se, nesta faixa etária, à redução da funcionalidade e maior dependência na realização das atividades de vida diária (AVDs)20-21.

As possíveis causas que contribuem para essa redução podem oscilar entre a falta de interesse, dificuldade de acesso, falta de energia, dores nas articulações, resistência em conhecer novas pessoas e participar de atividades em grupos 15. Outro fator que merece destaque é a diminuição da massa muscular, decorrente do processo de envelhecimento e consequentemente redução da força física16-17 e aptidão cardiorrespiratória, resultando em limitação da capacidade fisiológica para o exercício21. Mas ao mesmo tempo, a redução da AF também é responsável pela deterioração das capacidades físicas 18-19,22.

No último censo populacional, a taxa de analfabetismo brasileira para idosos foi de $26,2 \% 23$,valor semelhante ao presente estudo, no qual idosos com menos de 4 anos de escolaridade representaram juntos, $42,1 \%$. As diferenças no nivel de alfabetização refletem as desigualdades sociais do início do século, que bloquearam o acesso à escola para os pobres e as mulheres24. Por outro lado, a escolaridade e a aposentadoria são dois aspectos do perfil desses idosos que chamam atenção, pois sugerem que maior acesso a informação e maior disponibilidade de tempo está associada a maior prática de atividade física entre os idosos 23 .

No presente estudo, percebe-se um maior número de idosos casados e uma associação significativa desses idosos com a prática irregular de atividade física $(82,1 \%$ ). Esse fenômeno pode ser explicado pelo aumento progressivo na expectativa de vida da população e pela característica de relações conjugais longas e duradouras nessa faixa etária. Os indivíduos não casados às vezes apresentam menos tempo para cuidar de sua saúde devido as funções familiares que impedem desta busca a prática de atividade física25. Observou-se também que a maioria recebe de 1 a 2 salários mínimos, sendo esta renda maior do que aquela encontrada em outros estudos.

A atividade física muda para melhor a vida do idoso, não só as condições físico-motoras e a saúde geral26-27, mas, também, porque ocasiona maior fortalecimento das redes de suporte sociais, promovendo mudanças na vida cotidiana, como busca de melhoria da qualidade de vida28. Aanálise de componentes não fornece medidas de associação estatisticamente significativa entre as variáveis de saúde e o nível de atividade física, porém, segundo o presente estudo, o grupo de idosos muito ativos apresentou-se com melhor percepção de saúde, além de ausência tanto de doenças quanto de quedas nos últimos 6 meses e eram praticantes de atividade física há mais de 5 anos. Por outro lado, 0 grupo com prática irregular também apresentou boa percepção de saúde, ausência de doenças e ausência de quedas, resultados diferentes dos encontrados em um estudo29 no quais idosos com baixo nível de atividade física apresentavam percepção negativa de saúde.

Outro estudo30 avaliou a influência do NAF em relação à probabilidade de quedas, desequilíbrio e dificuldades na realização das AVDS. O grupo praticante de atividade física apresentou melhor pontuação nos domínios de equilibrio e marcha, além do escore total e da maior proporção dos idosos com independência nas AIVDs e ABVDS, ou seja, com menor probabilidade de queda e com maior nível de independência em relação ao grupo controle não praticante.

A prática de exercício físico prepara o idoso para uma vida autônoma e independente31. Os idosos do grupo mais ativo do presente estudo possuem maior independência para realização de ABVD e AIVD. Esses achados estão de acordo com estudos epidemiológicos onde evidenciam que o envelhecimento ativo tem relação direta com a capacidade funcional do idos031-34. Entretanto, esse mesmo grupo de idosos apresentava fraqueza muscular tanto em MMSS quanto em MMII, o que pode ser explicado pelo tipo de atividade física realizado por eles nas ATIs. E, apesar de constatada essa fraqueza, ela não interferiu negativamente na independência funcional dos idosos. 
Neste estudo, diferente de outros35-36, não foi observada relação entre funcionalidade e atividade física. Possivelmente, esses achados estão relacionados ao fato de que em ambos os grupos a maioria dos idosos são completamente independentes para as ABVDs e AIVDs, respectivamente. Esse achado, em particular, sugere dois questionamentos: o sedentarismo pode estar relacionado a outros fatores que não levam necessariamente a uma pior funcionalidade ou, ainda, o sedentarismo está mais associado a menor capacidade cognitiva e menor independência para AIVD, como encontrado na literatura 37.

No presente estudo não foi observada uma relação direta entre NAF, capacidade funcional e força muscular. Porém, quando comparada apenas capacidade funcional e força muscular dos idosos, notou-se uma correlação positiva $(p<0,05)$ das ABVDs com as AIVDs $(r=0,26)$ e com o teste de sentar e levantar $(r=0,25)$, resultado que indica uma fraca relação entre 0 aumento da capacidade funcional e 0 aumento da força muscular de MMIl; encontrou-se também uma correlação significativa entre o teste de flexão de braços e o teste sentar e levantar $(r=0,44)$, evidenciando uma relação moderada entre 0 aumento da força muscular de MMII e MMSS.

Uma possível explicação para esses achados é o tamanho e composição da amostra, que impossibilitou a generalização dos achados para a população geral e inferências mais robustas. Todos os idosos participaram do grupo de ginástica, por isso, não foi possível comparar a qualidade de vida entre os idosos praticantes e não praticantes de atividade física.

Outra limitação deste estudo diz respeito à complexidade do processo de envelhecimento ativo, juntamente com o desenho transversal do estudo que impossibilita a identificação da precedência temporal dos fatores estudados, comprometendo as evidências de relação causal.

Em relação à interpretação dos resultados, as informações derivadas dos testes utilizados e questionários são autorreferidos e, portanto, podem sofrer influência de funções cognitivas, culturais, da linguagem utilizada e da escolaridade. Em contrapartida, as medidas subjetivas fornecem informações valiosas sobre a qualidade e expectativa de vida dos idosos. Estudos epidemiológicos de base populacional adquirem importância nesse cenário por possibilitarem a identificação dos determinantes e dos fatores etiológicos do envelhecimento 38. Essa experiência se faz necessária uma vez que os idosos estão sujeitos a inúmeras variações e, futuramente, poderá contribuir para o conhecimento mais profundo do processo de envelhecimento da população.

Apesar das contribuições deste estudo, algumas limitações precisam ser apontadas. Ressalta-se também a ausência de um grupo de idosos que não praticam exercícios físicos, bem como idosos que praticam outros tipos de exercício, a fim de comparar os dados. Primeiramente, foram avaliados idosos de apenas uma cidade brasileira, o que não representa a realidade de idosos do país. No entanto, a amostra pode ser considerada relevante porque foram analisados idosos de Maringá, localizado no noroeste do Paraná, município pioneiro das ATIs que em 2006 implantou e serviu de modelo para todo o Brasil.

Outra limitação se refere ao design utilizado para analisar as associações entre as variáveis, uma vez que os resultados são baseados em dados de corte transversal, não permitindo inferências de causalidade. Dessa forma, futuras investigações devem continuar a explorar as associações entre tais variáveis, analisando idosos do sexo masculino, de outras regiões do país e com idade mais avançada (80 anos ou mais), bem como um acompanhamento dessa população em estudos longitudinais, a fim de compreender a relação causal entre as variáveis analisadas.

Considerações Finais

Neste estudo, tanto os idosos mais ativos quanto os menos ativos apresentaram bons níveis de independência funcional, entretanto aqueles com maior capacidade funcional apresentaram também maior força muscular de membros inferiores, e aqueles com maior força em membros inferiores apresentaram também maior força em membros superiores. Sugerindo que o nível de atividade física não seja fator determinante isolado para o nível de independência funcional, pois o mesmo engloba outros fatores, tais como força muscular, variáveis sociodemográficas e aspectos subjetivos não priorizados no presente estudo.
Referências

1. Vasconcelos APSL, Cardozo DC, Lucchetti ALG, Lucchetti G. Comparison of the effect of different modalities of physical exercise on functionality and anthropometric measurements in community-Dwelling older women. J Body Mov Ther. 2016;2(10):67-75 2. Duarte $L M N$. O processo de institucionalização do idoso e as territorialidades: espaço como lugar? Est Interdis Sobr Envelhec. 2014;19(1):201-17.

3. Fechine BRA, Trompieri N. O processo de envelhecimento: as principais alterações que acontecem com o idoso com o passar dos anos. Inter Science Place. 2015;1(20):106-32. 4. Rosa RS, Bianchi PDA, Hansen D, Monschau BT. Alterações fisiológicas da força muscular respiratória decorrente do envelhecimento sobre a funcionalidade de idosos. Fisioter Bras. 2014;15(1):16-21

5. Silva CM, Gurjão ALD, Ferreira L, Gobbi LTB, Gobbi S. Efeito do treinamento com pesos, prescritos por zona de repetições máximas, na força muscular e composição corporal em idosas. Rev Bras Cineantrop Desemp Hum. 2006:8(4):39-45.

6. Assis EL, Rabelo HT.Percepção da capacidade funcional de mulheres idosas praticantes de hidroginástica. Movimentum. 2006;1(1):1-16.

7. Souto PPC, Bandeira TF, Viana FP, Sandoval RA. Força muscular de membros superiores e inferiores: estudo correlacional comparativo entre grupos de idosas. Estudos. 2010:37(1):83-100.

8. Ferreira L, Barbosa TD, Gobbi S, Arantes LM. Capacidade Funcional em mulheres jovens e idosas: projeções para uma adequada prescrição de exercícios físicos. Rev Educ Fís UEM. 2008:19(3):403-12.

9. Pícoli TS, Figueiredo LL, Patrizzl LJ. Sarcopenia e envelhecimento. Fisioter Mov. 2011:24(3):455-62.

10. Oliveira DV, Batilani JR, Bertolini SMMG, Carvalho EA. Caracterização das atividades físicas, condições de saúde e hábitos de vida dos idosos usuários da academia da terceira idade. Rev Cient Jopef. 2013:15(1):1-17.

11. Maciel MG. Atividade física e funcionalidade do idoso. Motriz. 2010:16(4): 1024-32.

12. Mazo GZ, Benedetti TR. Bertoldo. Adaptation of the international physical activity questionarie for the elderly. Rev Bras Cineantr Desemp Humano. 2010;12(6):480-4.

13. Rickli RE, Jones CJ. Testes de aptidão física para idosos. Barueri, SP: Manole, 2008

14. Borges MRD, Moreira AK. Influências da prática de atividades físicas na terceira idade: estudo comparativo dos níveis de autonomia para o desempenho nas AVDs e AIVDs entre idosos ativos fisicamente e idosos sedentários. Motriz. 2009:15(3):562-73.

15. Crombie IK, Irvine L, Williams B. Why older people do not participate in leisure time physical activity: a survey of activity levels, beliefs and deterrents. Age Agein. 2004;33(3):287-92.

16. Evans W. Skeletal muscle loss: cachexia, sarcopenia, and inactivity. Am J Clin Nutr. 2010;91(11):11-7.

17. Muscaritoli M, Anker SD, Argilés J, Aversa Z, Bauer JM, Boirie Y, et al. Consensus definition of sarcopenia,

cachexia and pre-cachexia: Joint document elaborated by Special Interest 
Groups (SIG) "cachexia-anorexia in chronic wasting diseases" and "nutrition in geriatrics". Clin Nutr 2010;29(2):154-9.

18. Booth FW, Zwetsloot KA. Basic concepts about genes, inactivity and aging.

Scand J Med Sci Sports. 2010:20(1):1-4.

19. Chipperfield JG. Everyday physical activity as a predictor of late-life mortality. Gerontologist 2008;48(3):349-57.

20. Frisard MI, Fabre JM, Russell RD. Physical activity level and physical functionality in nonagenarians compared to individuals aged 60-74 years. J Gerontol Biol Sci Med Sci. 2007;62(7):783-8.

21. Tribess S, Virtuoso-Júnior JS, Petroski EL. Fatores associados à inatividade física em mulheres idosas em comunidades de baixa renda. Rev Salud Publica 2009;11(1):39-49.

22. Lord S, Chastin SFM, Mclnne L, Little L, Brigges P, Rochester L. Exploring patterns of daily physical and sedentary behaviour in community-dwelling older adults. Age Ageing. 2011;40(1):205-10

23. Datasus [online]. Departamento de Informática do SUS. Ministério da Saúde. Taxa de analfabetismo- B.1. 2011. Disponível em: http://tabnet.datasus.gov.br/cgi/tabcgi. exe?idb2011/b01a.def

24. Araújo CL, Manucussi e Faro AC. La práctica de actividad física en adultos mayores del Valle del Paraíba, São Paulo, Brasil. Enferm Global. 2012;11(28):204-12.

25. Pereira JRP, Okuma SS. O perfil dos ingressantes de um programa de educação física para idosos e os motivos da adesão inicial. Rev Bras Educ Fís Esporte. 2009;23(4):319-34. 26. Taaffe DR, Irie F, Masaki KH, Abbott RD, Petrovitch H, Ross GW, et al. Physical activity, physical function, and incident dementia in elderly men:The Honolulu-Asia Aging Study. $J$ Gerontol A Biol Sci Med Sci. 2008;63(5):529-35.

27. Dumith SC.Atividade física e sedentarismo: diferenciação e proposta de nomenclatura. Rev Bras Ativ Fís Saúde. 2010;15(4):253-4.

28. Sui X, LaMonte MJ, Laditka JN, Hardin JW, Chase N, Hooker SP et al. Cardiorespiratory fitness and adiposity as mortality predictors in older adults. JAMA. 2007;298(21):2507-16.

29. Boscatto EC, Duarte MFS, Barbosa AR. Nível de atividade física e variáveis associadas em idosos longevos de Antônio Carlos, SC. Rev Bras Ativ Fis e Saúde. 2012:17(2):132-6.

30. Figliolino JAM, Morais TB, Berbel AM, Dal Corso S. Análise da influência do exercício físico em idosos com relação a equilíbrio, marcha e atividade de vida diária. Rev. Bras. Geriatr. Gerontol. 2009:12(2):227-38.

31. Gordia PA, Quadros BMT, Vilela Junior VBG, Souza AE, Cabral C, Morais BT, et al. Comparação da qualidade de vida de mulheres idosas praticantese não praticantes de exercício físico. Educ Fís Esp. 2007;11(106):1-14.

32. Tavares DMS, Dias FA. Distribuição espacial de idosos de acordo com menores escores de qualidade de vida. Texto Contexto Enferm. 2011;20(1):205-13.

33. Guimarães ACA, Scotti AV, Soares A, Fernandes S, Machado Z. Percepção da qualidade de vida e da finitude de adultos de meia idade e idoso praticantes e não praticantes de atividade física. Rev Bras Geriatr Gerontol. 2012;15(4):661-70.

34. Ferreira OGL, Maciel SC, Costa SMG, Silva AO, Moreira MASP. Envelhecimento ativo e sua relação com a independência funcional. Texto Contexto Enferm. 2012:21(3):513-8.

35. Tavares DMS, Dias FA. Capacidade funcional, morbidades e qualidade de vida de idosos. Texto Contexto Enferm. 2012;21(1):112-20.

36. Farias RG, Santos SMA. Influência dos determinantes do envelhecimento ativo entre idosos mais idosos. Texto Contexto Enferm. 2012;21(1):167-76.

37. Campos ACV, Cordeiro EC, Rezende GP, Vargas AMD, Ferreira EF. Qualidade de vida de idosos praticantes de atividade física no contexto da estratégia saúde da família. Texto Contexto Enferm, Florianópolis, 2014;23(4): 889-97

38. Fiedler MM, Peres KG. Capacidade funcional e fatores associados em idosos do Sul do Brasil: um estudo de base populacional. Cad Saúde Pública. 2008;24(2):409-15. 